\title{
Exocytotic fusion pore under stress
}

\author{
Helena Haque Chowdhury ${ }^{1,2}$ and Robert Zorec ${ }^{1,2, *}$ \\ ${ }^{1}$ Laboratory of Cell Engineering, Celica Biomedical, 1000 Ljubljana, Slovenia. \\ ${ }^{2}$ Laboratory of Neuroendocrinology - Molecular Cell Physiology, Institute of Pathophysiology, University of Ljubljana, Medical Facul- \\ ty, 1000 Ljubljana, Slovenia. \\ * Corresponding Author: \\ Robert Zorec, Laboratory of Neuroendocrinology - Molecular Cell Physiology, Institute of Pathophysiology, University of Ljubljana, \\ Medical Faculty, 1000 Ljubljana, Slovenia.Tel:+386 1543 7081; Fax:+386 1543 7036; E-mail: robert.zorec@mf.uni-lj.si
}

\begin{abstract}
Exocytosis is a universal process of eukaryotic cells, consisting of fusion between the vesicle and the plasma membranes, leading to the formation of a fusion pore, a channel through which vesicle cargo exits into the extracellular space. In 1986, Rand and Parsegian proposed several stages to explain the nature of membrane fusion. Following stimulation, it starts with focused stress destabilization of membranes in contact, followed by the coalescence of two membrane surfaces. In the next fraction of a millisecond, restabilization of fused membranes is considered to occur to maintain the cell's integrity. This view predicted that once a fusion pore is formed, it must widen abruptly, irreversibly and fully, whereby the vesicle membrane completely integrates with and collapses into the plasma membrane (full fusion exocytosis). However, recent experimental evidence has revealed that once the fusion pore opens, it may also reversibly close (transient or kiss-and-run exocytosis). Here, we present a historical perspective on understanding the mechanisms that initiate the membrane merger and fusion pore formation. Next, post-fusion mechanisms that regulate fusion pore stability are considered, reflecting the state in which the forces of widening and constriction of fusion pores are balanced. Although the mechanisms generating these forces are unclear, they may involve lipids and proteins, including SNAREs, which play a role not only in the pre-fusion but also post-fusion stages of exocytosis. How molecules stabilize the fusion pore in the open state is key for a better understanding of fusion pore physiology in health and disease.
\end{abstract}

doi: $10.15698 /$ cst2020.09.230

Received originally: 15.05 .2020

in revised form: 19.07.2020,

Accepted 29.07.2020,

Published 10.08.2020.

Keywords: exocytosis, fusion pore, SNARE proteins, secretory vesicle, fusion pore stability.

\section{Abbreviatons:}

dnSNARE - dominant-negative domain of synaptobrevin 2 protein peptide; SNARE - soluble NSF-attachment protein receptors.

\section{INTRODUCTION}

Although it is more than seven decades since Sir Bernard Katz and colleagues started to investigate synaptic vesicle fusion with the plasma membrane at the end-plate (the neuromuscular junction), progress in understanding this ubiquitous process of eukaryotic cells has been slow. The original observation of "biological noise" (Fig. 1) at the stimulated neuromuscular junction, represented by miniature end-plate potentials [1], already recorded in 1948 at the skeletal muscle membrane [2], was instrumental for the formulation of the "quantal theory of neurotransmitter release". This was recognized widely by awarding the Nobel Prize in 1970 to Sir Bernard Katz, Ulf von Euler and Julius Axelrod, "for their discoveries concerning the humoral transmitters in the nerve terminals and the mechanism for their storage, release and inactivation" [3]. In his Nobel
Laureate lecture, Sir Bernard Katz outlined the events that regulate neurotransmitter release: depolarization opens specific "calcium gates" in the terminal axon membrane, leading to an influx of calcium ions to initiate the "quantal release reaction". Based on biochemical and structural data available at that time, he also considered the hypothesis "that the quanta of transmitter molecules are enclosed within synaptic vesicles which frequently collide with the axon membrane, and that calcium causes attachment and local fusion between vesicular and axon membranes" [4]. He postulated a structure at the plasmalemma where vesicles frequently collide, depicted as black dots in Fig. $\mathbf{2}$.

A further step towards understanding the nature of events that occur during the merger of the vesicle membrane with the plasmalemma was the consideration that this process consists of distinct steps [5]. (i) Stable mem- 

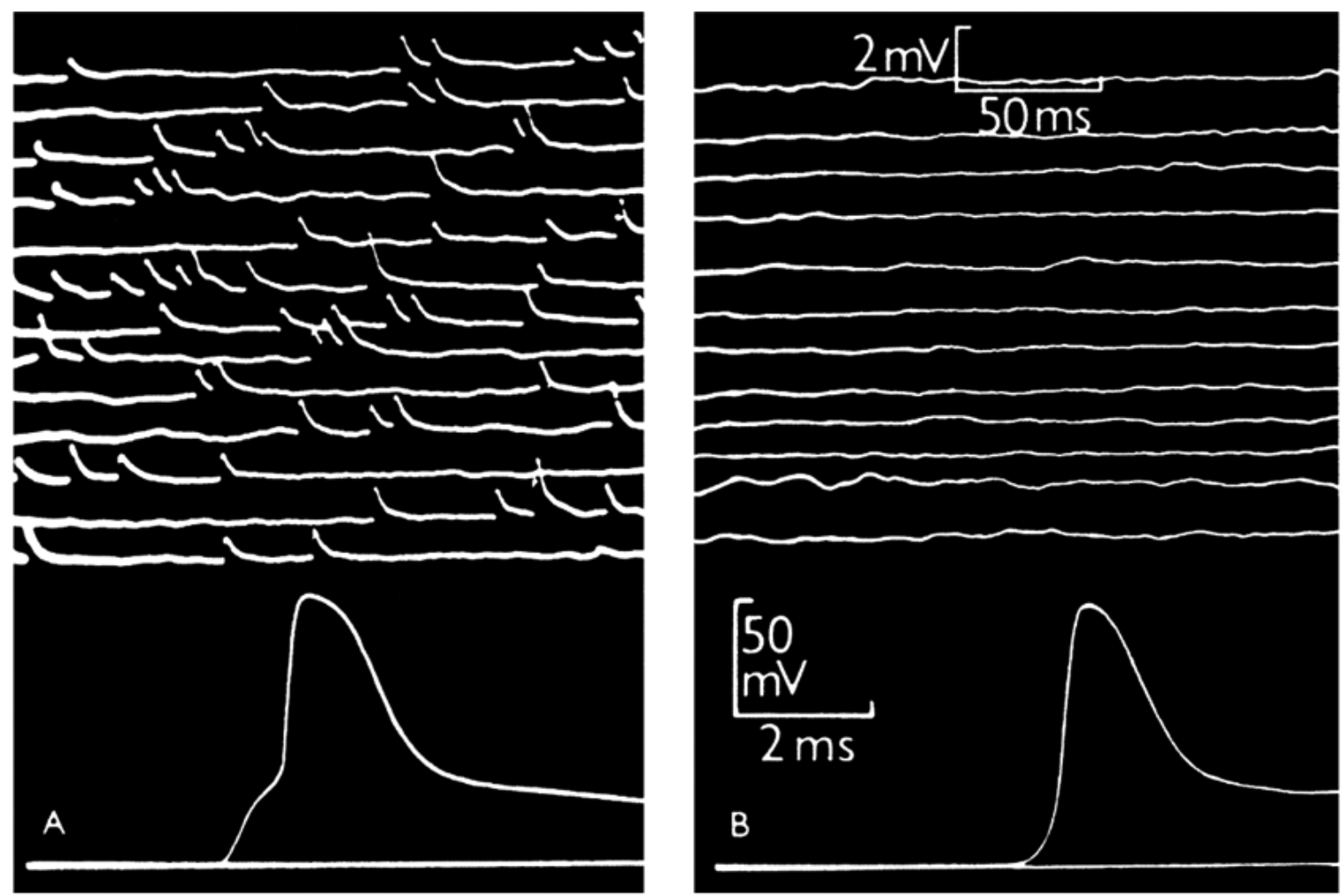

FIGURE 1: Intracellular recording from single muscle fibre of frog. (A) At the motor end-plate. The upper part shows spontaneous miniature end-plate potentials, which are localized at a junction and arise from sudden discharge by a motor nerve ending of packets of acetylcholine, each containing thousands of molecules. The lower part shows a single response to a nerve impulse, which was started by electric shock at the beginning of the trace; the first step of the response is large end-plate potential resulting from synchronous delivery of a few hundred packets of acetylcholine, this leading to full size action potential. (B) Traces recorded in the same muscle fibre, $2 \mathrm{~mm}$ away from the endplate. The upper part shows much attenuated and barely recognizable residues of miniature end-plate potentials. The lower part shows propagated action potential, delayed by conduction over $2 \mathrm{~mm}$ distance and not preceded by the end-plate step. From [1]

brane apposition: Rand and Parsegian argued that most membranes do not fuse, however, when membrane fusion occurs, there are exceptional, strictly controlled conditions to allow specific membrane pairs to merge. (ii) Triggering of membrane fusion was considered to be associated with an increase in intracellular calcium. (iii) Contact fusion was considered to involve a closer functional merger of membranes, closer than the stable apposition achieved by most membranes. (iv) Focused destabilization was considered in such a way that the structure of the contacting membranes must destabilize, and that destabilization must be restricted to the contact area to ensure that the fusion of membranes is "leakless," i.e. that the correct aqueous compartments mix. (v) Membrane coalescence: the destabilized structure was considered to lead to the coalescence of two membrane surfaces. (vi) Restabilization: the fused membrane was thought to restabilize quickly to maintain the integrity of the membrane and the cell. Importantly, Rand and Parsegian noted that stages (iv), (v), and (vi), and perhaps (iii), appear extremely rapidly (in microseconds), even if the events leading to them are slow. This latter view prompted the idea that once the fusion pore is formed, it must widen abruptly and fully. This mode of exocytosis is often termed full fusion (irreversible) exocy- tosis, meaning that the vesicle membrane completely integrates with and collapses into the plasma membrane and is retrieved only by regulated endocytosis.

In contrast to this mode of exocytosis, recent data revealed that the fusion pore may enter a stable, dynamically regulated state, with a diameter ranging between subnanometres to several hundred nanometres $[6,7]$. These fusion pores can then undergo either reversible constriction/closure or can widen again, consistent with the original observation that synaptic vesicles fuse with, and re-form from, the membrane of the nerve terminal during stimulation and that the re-formed vesicles can store and release transmitter [8]. This mode of exocytosis was named as kiss-and-run exocytosis [9]. This terminology has been considered in part by biophysical patch-clamp experiments to monitor membrane currents and membrane capacitance, a parameter linearly related to changes in the membrane area. This technique permits direct determination of the fusion pore opening and closing [10] and has been used in many secretory cells, including chromaffin, mast, pancreatic, plant and pituitary cells [10-17], where it has revealed that in addition to full fusion, the fusion pore, once open, can reversibly close or constrict (Fig. 3). These observations established that the fusion pore opening is a 

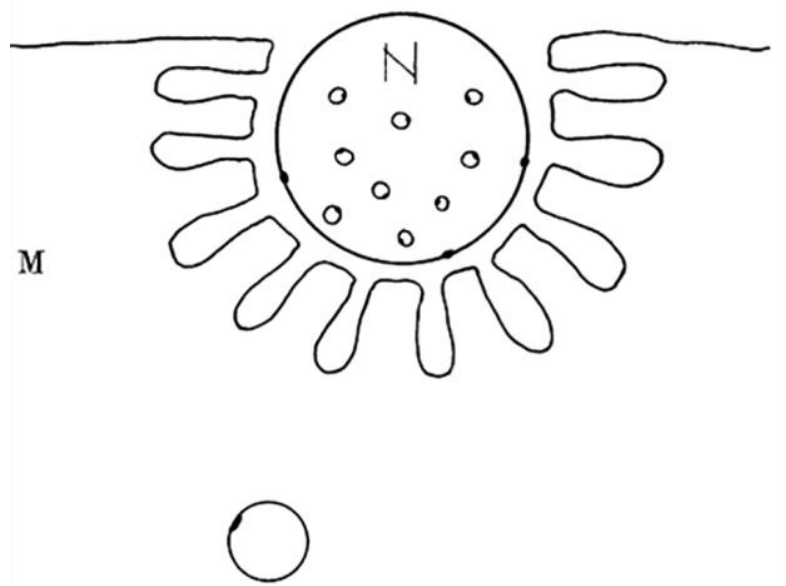

$b$
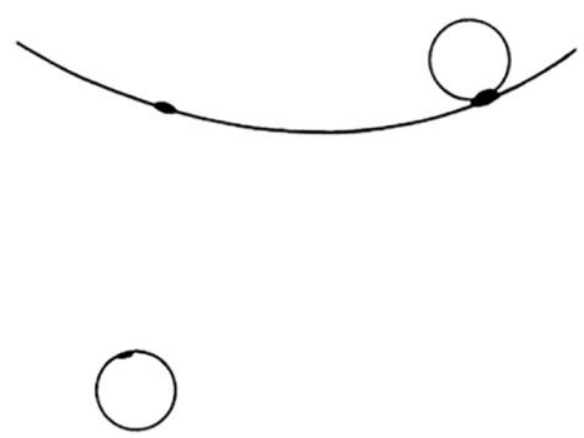

$c$

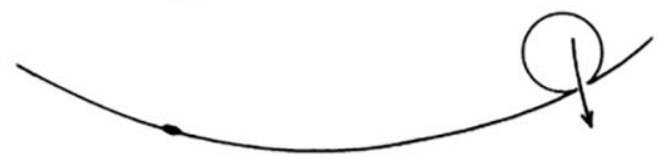

FIGURE 2: Diagram explaining quantal secretion of neurotransmitters by exocytosis of synaptic vesicles. Reaction molecules (fusion proteins) are indicated by black dots on vesicular and nerve membranes; a nerve impulse greatly increases the number of reactive sites in the terminal membrane by allowing calcium ions to penetrate it. $\mathrm{N}$, motor nerve terminal; $\mathrm{M}$, end-plate region of a muscle fibre. This scheme was presented by J. del Castillo and B. Katz at a Symposium at Gif-sur-Yvette in July 1955 [73].

real biological phenomenon where the fusion pore conductance, a measure of how narrow is the fusion pore, can be determined under different physiological conditions. By studying giant secretory vesicles, currents through the open fusion pore were measured [18]. Simultaneous electrical and optical measurements revealed that the formation of the fusion pore precedes vesicle swelling [19]. Reversible closure (transient opening) of the fusion pore may occur several times, and it was observed in different cell types including chromaffin and pituitary cells, plant protoplasts and astrocytes [6,13,17, 20-22]. Hence, it is highly unlikely that the same vesicle "runs" away from the plasmalemma each time the fusion pore closes. Therefore, this form of exocytosis was termed reversible or transient fusion pore opening or "reversible or transient exocytosis" $[6,17]$. The fusion pore opening can result in a very narrow pore diameter, smaller than the actual molecular size of the vesicle cargo, thus this cargo cannot be secreted to the cell exterior. In this case, the mode of exocytosis was termed "unproductive exocytosis" [23]. Fig. 3E indicates transitions between the intermediate fusion stages of an exocytotic vesicle. The initial narrow fusion pore can reversibly widen and return to a narrow or even a closed state, however at some point in time it may also transit to a fully wide state (full fusion).

Transitions between the stages that the vesicle passes during membrane merger appear to depend on vesicle size $[7,24,25]$, and it is generally acknowledged that these transitions are regulated by proteins [7, 26], which may also form the fusion pore walls [27-29]. The fusion pore was also modelled to be exclusively lined by lipids [30]; however, it is more likely to be a mixture of proteolipids $[31,32]$. Although it is experimentally difficult to record single-vesicle fusion in synapses, which are specialized for fast signalling, and the vesicles are relatively small, it appears that fusion pores of these vesicles predominantly exhibit fully open fusion pores, with a small fraction of events exhibiting transient fusion pore openings [33-35]. In these vesicles, the opening of the fusion pore follows the calcium trigger in a millisecond and is considered to be associated with the SNARE (soluble NSF-attachment protein receptors) protein complex, which is thought to initiate exocytotic membrane fusion [36]. In the next sections, we highlight the nature of pre- and post-fusion mechanisms of the vesicle membrane merger with the plasmalemma.

\section{PRE-FUSION MECHANISMS}

The initial stages of membrane fusion were considered to consist of focused destabilization, whereby a spatially restricted structure of the contacting membranes must destabilize to allow membrane coalescence [5]. Based on these predictions, where the proximity between membranes should be much tighter than the ordinary membrane-to-membrane distance, typically determined in part by the repulsion of negatively charged membrane surfaces [5], a suitable mechanism may involve proteins that span two membranes and interact to bring the two membranes closer. Among many recognized candidates, proteins termed SNAREs have received attention. Their discovery was part of the 2013 Nobel Prize in Physiology or Medicine, awarded to James E. Rothman, Randy W. Schekman and Thomas C. Südhof "for their discoveries of machinery regulating vesicle traffic, a major transport system in our cells" [37]. Whereas the coworkers of T.C. Südhof discovered signals that coordinate the timing of when the synaptic vesicles release their cargo in neurons, the coworkers in the lab of R. Schekman described a set of genes required for vesicle transport. The research from the group of J.E. Rothman studied the SNAREs, a group of three proteins: vesicle-associated membrane protein (VAMP)/Synaptobrevin, synaptosome-associated protein of $25 \mathrm{kDa}$ or $23 \mathrm{kDa}$ (SNAP-25 or SNAP-23) and syntaxin. These three interacting SNARE proteins, localized to the presynaptic region, had previously been identified and 
(a) (i)

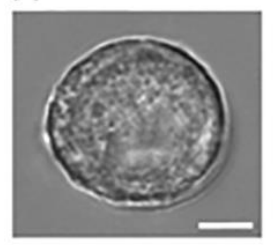

(ii)

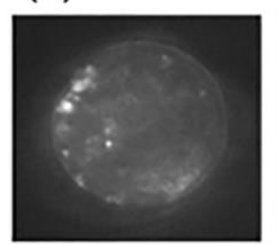

(iii)

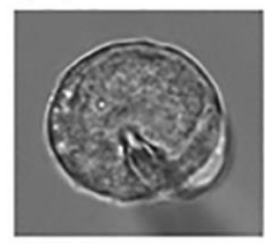

(b)

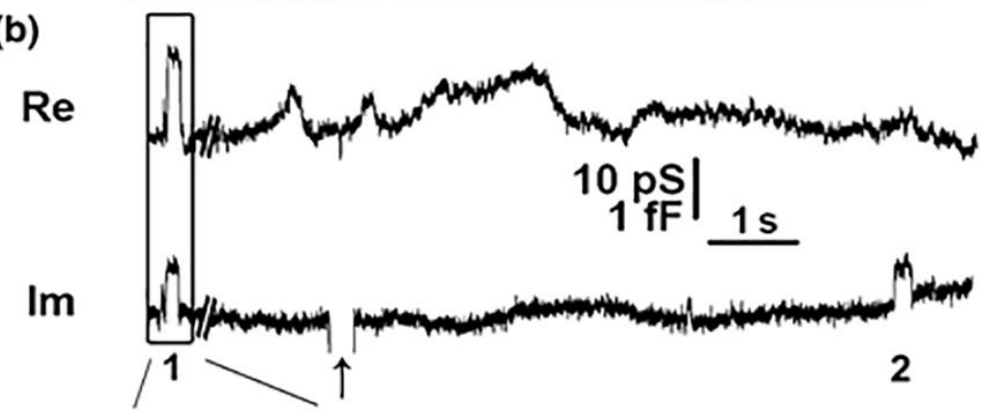

(c)

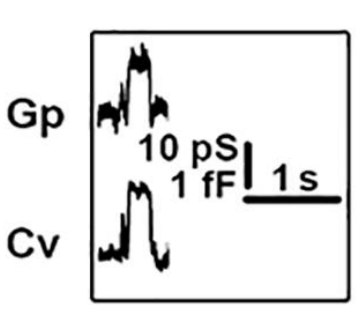

(d) $\operatorname{Re}$

(e)

Narrow fusion pore
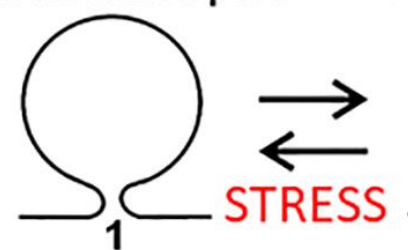

Wide fusion pore
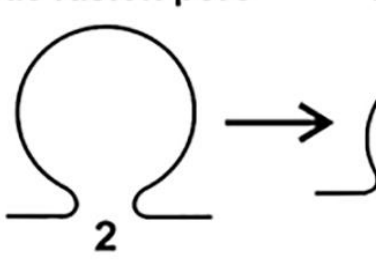

2

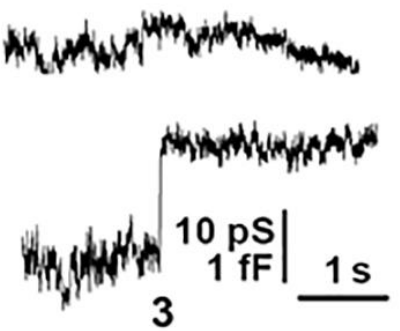

Full fusion

studied by researchers (including Kimio Akagawa, Richard Scheller, Reinhard Jahn and Pietro de Camilli), but their function was largely unclear. These proteins were found to be ideally located across the vesicle and the plasmalemma; VAMP/Synaptobrevin is a vesicle protein, whereas SNAP25 and syntaxin were found at the plasma membrane. This enabled the generation of the SNARE hypothesis proposing that target and vesicle SNAREs (t-SNAREs and v-SNAREs) are engaged in vesicle fusion through several sequential steps, including hemifusion, an early stage of membrane coalescence according to Rand and Parsegian. This stage was visualized in larger, experimentally more accessible vesicles [38] and is likely playing a role in vesicle docking (anchoring to the specific sites, predicted already by Sir Bernard Katz; Fig. 2). These initial steps involve the activation of a local mechanical stress to promote membrane fusion, leading to the merger of the vesicle and the plasma membranes. This interpretation, especially the local mechanical stress during focal apposition of fusing membranes, was influenced by bulk biochemical studies, which revealed that the ternary SNARE complex (an association of the three types of SNARE molecules) is a thermally very
3
FIGURE 3: Cell-attached membrane capacitance $\left(C_{m}\right)$ measurements on pituitary lactotrophs show transient (reversible) and full fusion exocytotic events. (A) Lactotrophs co-transfected with hyperpolarization-activated and cyclic nucleotide-gated 2 pDNA and EGFP pDNA were used for the $C_{m}$ measurements in the cell-attached configuration [12]. (Ai-Aiii) All measurements were performed only on lactotrophs exhibiting EGFP fluorescence, as shown for the same cell under transmitted light (Ai), under epifluorescence (Aii), and during the measurement (Aiii). Scale bar, $3 \mu \mathrm{m}$. (B) An epoch from a representative recording showing the reversible steps in the Im part of the admittance trace. Reversible events in Im, which likely represent transient exocytotic events (transient fusion pore opening), either exhibited projections to the Re part of the admittance trace (1) or not (2). The arrow points to the calibration pulse in $I m$, which was triggered manually to ensure the correct phase angle settings. (C) Calculated vesicle capacitance (Cv) and fusion pore conductance $(G p)$, a measure of fusion pore geometry, for all reversible events with projections [74]. (D) A representative example of irreversible upward steps in the $\mathrm{Im}$ trace and the corresponding Re trace (3), which likely denotes a full fusion exocytotic event. (E) A scheme of the stages that a vesicle must pass to completely fuse with the plasma membrane: from narrow fusion pore formation (1), to widening of the fusion pore (2), and finally to full fusion of the vesicle membrane and the plasma membrane (3). From Ref. [74]. stable structure [39]. This led to the concept that once the ternary SNARE complex is formed, it may not be easily disassembled unless special enzymes with provision of energy in the form of ATP are in place [40]. Hence, this stable SNARE complex can be considered a molecular nucleus generating a focal mechanical stress, predicted by Rand and Parsegian in 1986. In the next stages, this focal structure was predicted to enable vesicle cargo discharge in an all-or-none-fashion, as envisioned by the "quantal release of neurotransmitter" considered by Sir Bernard Katz.

Anchoring of the SNARE proteins to the specific sites at the plasma membrane is related to membrane rafts [41], structures with enriched amounts of cholesterol, the most common steroid in humans and a major constituent of the cell membrane, which affects membrane fusion [42]. Although many experiments addressed this topic in the past [43-47], direct demonstration of how cholesterol affects distinct stages of regulated exocytosis, especially the dynamics of single fusion pore gating is still unclear. It is known that an increase in membrane cholesterol increases the probability of a vesicle fusing with the plasma membrane [48]. These findings are consistent with the view that 
negative curvature of cholesterol promotes the first steps of the membrane merger pathway $[45,49,50]$, the formation of restricted sites in the membrane, where focused destabilization, according to Rand and Parsegian, can be initiated. However, the question was raised as to whether the increased curvature due to enhanced cholesterol content in membrane rafts is sufficient to represent a native mechanism of membrane fusion, without any additional presence of proteins such as SNAREs [50].

The presence of membrane rafts, and especially the anisotropic negative curvature shape of cholesterol, has been viewed as functionally affecting the ability to shape the membrane curvature locally, which may influence the fusion pore formation and its stabilization [24, 45]. However, a less explored mechanism of the role of membrane rafts in membrane fusion mechanisms is that represented by the generation of tension between intermembrane domains with heterogeneous structure [51, 52], which may play a role in post-fusion mechanisms.

\section{POST-FUSION MECHANISMS}

Recently accumulated evidence that the fusion pore may enter into a relatively stable but dynamically regulated state $[6,7]$ indicates that there are forces that control fusion pore opening and closure. When these forces are balanced, the fusion pore exhibits a stable fusion pore diameter. Although the mechanisms of fusion pore stabilization are unknown, vesicle size itself and several proteins, including actin, syntaxin, synaptotagmin, Munc 18-1, dynamin, CAPS and others appear to play a role [7, 24-26, 29, 53-56].

The cortical actin cytoskeleton was considered a barrier for exocytotic vesicle delivery to the plasma membrane [57-59]. Subsequently, however, actin was found to be required for regulated exocytosis [60], in particular for widening of fusion pores with diameters exceeding $100 \mathrm{~nm}$ [7], an intermediate structure through which vesicle membrane transfer to the plasmalemma is manifested as vesicle shrinkage [61-63], influenced by the osmotic pressure difference between the intracellular and the extracellular solution and actin-providing membrane tension to reel in the membrane of the fusing vesicles $[62,63]$. The transition of the membrane fusion pore with a completely widened diameter arguably results from competition between calcium-dependent activation of exocytotic machinery and dynamin-dependent fusion pore closure $[38,55]$.

Interestingly, SNARE proteins not only play a role in pre-fusion but also during post-fusion mechanisms. Indeed, when the function of the accessory SNARE protein Munc 18-1 was studied at the level of a single-vesicle interaction with the plasmalemma, using the high-resolution patchclamp capacitance technique, it was revealed that there are multiple sites where the SNARE complex controls the exocytotic intermediates [26]. In the study using endocrine pituitary cells, Munc 18-1 mutants were transfected into secretory cells to affect the interaction of Munc 18-1 with syntaxin1 (Synt1, R39C), with Rab3A (E466K), and Mint proteins (P242S). In comparison with wild-type Munc 18-1, the mutant Munc 18-1E466K increased the frequency of the unitary fusion events, consistent with the view that Rab3A protein, a small GTPase [64], facilitates vesicle docking at the plasma membrane. Whereas the other Munc 181 mutants (R39C and P242S) increased the fusion pore dwell-time, all the mutants stabilized the geometry of a narrow diameter fusion pore, indicating that transition of a narrow diameter fusion pore into a more widely or completely open one is hindered by all these mutants. Singlemolecule atomic force microscopy experiments revealed that wild-type Munc 18-1, but not Munc 18-1R39C, abrogates the interaction between synaptobrevin2 (Syb2, a vSNARE protein) and Synt1 binary trans-complexes [26]. Importantly, neither form of Munc 18-1 affected the interaction of Syb2 with the preformed binary cis-Synt1-SNAP25 complexes at the plasmalemma, revealing that Munc 18-1 performs a proofing function by inhibiting tethering of Syb2-containing vesicles solely to Synt1 at the plasmalemma and promoting vesicular tethering via Syb2 to the preformed binary cis-complex of Synt1-SNAP25 (Fig. 4A).

The studies using atomic force spectroscopy of single SNARE molecule interactions revealed the disassembly properties of the ternary SNARE complex to be in the time domain of 0.2 to $2 \mathrm{~s}$ [65], which is consistent with fusion pore kinetics $[22,26]$. Therefore, assembly and disassembly of the ternary SNARE complex that is influenced by SNARE accessory proteins, can take place not only at vesicle docking, which facilitates the vesicle membrane merger with the plasma membrane, but also at other later intermediate stages of exocytosis (Fig. 4B), such as the transient widening of the fusion pore and regulation of the fusion pore dwell-time, both processes leading to full fusion, a complete integration of vesicle membrane into the plasma membrane [66].

Fig. 4B depicts the stages that a vesicle undergoes to reach the capacity to fully integrate with the plasma membrane (full fusion). The last step in the sequence of transitions is indicated to be inhibited by the dominant-negative domain of synaptobrevin 2 protein peptide (dnSNARE). This peptide has been considered to block the formation of the ternary SNARE complex and was hence used to block vesicle-based exocytosis, especially in astrocytes, a type of neuroglial cells [67]. Although it is still debated whether gliotransmission, a process that depends on the rapid detection of synaptic activity by astrocytes, is present in vivo, ample evidence indicates that regulated exocytosis is present in astrocytes $[22,68]$ but with much slower kinetics [69] than in neurons. The paradigm of rapid transmitter release from glial cells to be present in vivo critically depends on experiments in transgenic mice expressing the dnSNARE peptide. However, the mechanism of action of this peptide at the level of a single vesicle was recently described [22], revealing that the action of this peptide is not at the pre-fusion stage, the stage of the formation of the SNARE complex, but at the stage when the fusion pore is already open [22]. Moreover, when botulinum neurotoxins cleaving Syb2 and SNAP-23 were transfected into cells, the frequency of unitary exocytotic events was reduced as expected if the formation of ternary SNARE complex is 

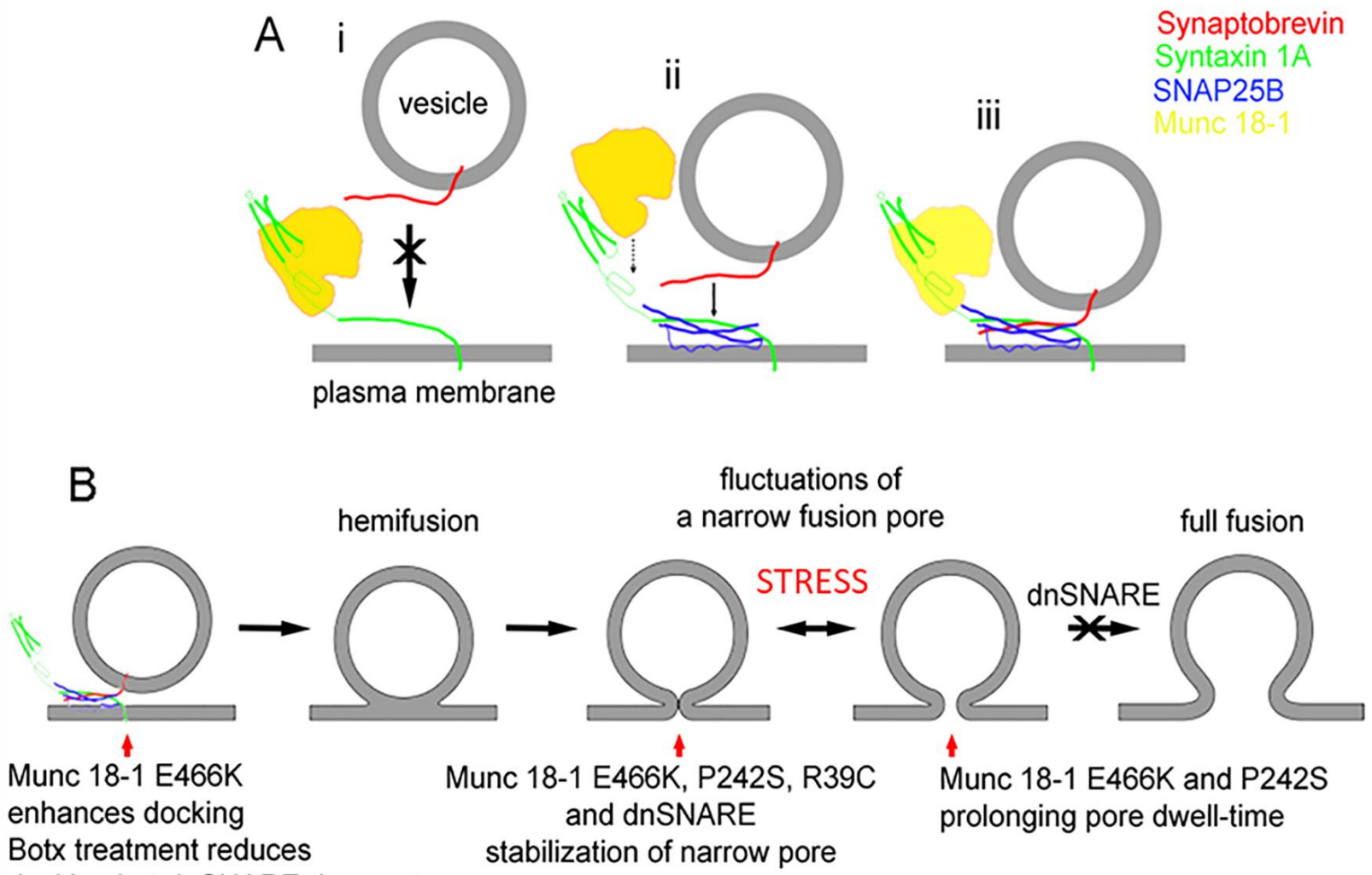

FIGURE 4: A diagram displaying the interaction of Munc18-1 an SNARE proteins along the stages of exocytosis. (A) The interaction between Munc18-1 and SNARE proteins, indicating that Munc18-1 favours the formation of the ternary SNARE complex where synaptobrevin binds preferably with the binary complex between syntaxin and SNAP25. (B) The stages from docking/priming, hemifusion state, leading to fusion pore formation and full fusion, with the action of respective Munc18-1 mutants and dnSNARE peptides. From Ref [26].

needed for the vesicle fusion with the plasma membrane. However, when the dnSNARE peptide was introduced into cells, the frequency of exocytotic events was unaffected, consistent with the view that dnSNARE peptide acts at the post-fusion stage of regulated exocytosis, constricting the pore to a very narrow diameter. This dnSNARE-mediated fusion pore stabilization in astrocytes [22], which is considered release unproductive [23], was observed in lactotrophs transfected with Munc 18-1 mutants [26].

Mutant SNARE-interacting proteins (Munc18-1, Rab3A, and Mints), as well as a dominant-negative synaptobrevin peptide dnSNARE, stabilize fusion pores with subnanometre diameter $[22,26]$, indicating that the SNARE complex in the "unzippered" state (i.e. SNARE proteins are unable to interact to form a complex) is unable to oppose the forces constricting the fusion pore, clearly a post-fusion mechanism. The nature of these forces is generally not known, but dynamin is a strong candidate here $[38,55]$. Moreover, lipids can also regulate the fusion pore [32], including phosphatidic acid produced by phospholipase D1. It has been shown that phosphatidic acid helps in recruiting and/or activating the exocytotic protein machinery. It also directly affects the membrane curvature and thus favours membrane rearrangements as required for membrane fusion [70]. A similar mechanism was previously proposed for phosphatidylinositol 4,5-bisphosphate (PIP2) [71] and for cholesterol [50]. In the latter case, as already discussed in the previous section, membrane rafts enriched with cholesterol may generate a force on the neighbouring membrane regions with heterogeneous membrane structure [52], and these forces may play a role in membrane fusion [42]. However, direct demonstration of such a role of cholesterol in membrane fusion has only recently been addressed [72].

\section{CONCLUSIONS}

In summary, regulated exocytosis is a multistage process. In the past, the stages that a vesicle passes during exocytosis were considered to occur abruptly at specific sites to minimize the leak of cytosolic components during membrane destabilization, hence the process was considered to be irreversible, ending in full fusion. Recent data obtained by monitoring vesicle fusion at the single-vesicle level revealed that fusion pores exhibit remarkable stabilization: not only when they are smaller than a nanometre but also at several hundred nanometres of opening. These stable fusion pore diameters reflect a balance between forces that open and close the fusion pore. Although proteins (especially SNARE proteins) are considered the major players in the regulation of fusion pore diameter, there is evi- 
dence that lipids, including cholesterol, play a role in preand post-fusion mechanisms. Therefore, the membranes engaged in fusion pore formation exhibit mechanical stress during initiation of the process but also at the stage when the pore is already open.

\section{ACKNOWLEDGMENTS}

The authors acknowledge the support from the Slovenian Research Agency for National core program Cell Physiology No P3 310.

\section{CONFLICT OF INTEREST}

No conflict of interest in connection with this manuscript is declared.

\section{COPYRIGHT}

(C) 2020 Chowdhury and Zorec. This is an open-access article released under the terms of the Creative Commons Attribution (CC BY) license, which allows the unrestricted use, distribution, and reproduction in any medium, provided the original author and source are acknowledged.

Please cite this article as: Helena Haque Chowdhury and Robert Zorec (2020). Exocytotic fusion pore under stress. Cell Stress 4(9): 218-226. doi: 10.15698/cst2020.09.230

13. Ales E, Tabares L, Poyato JM, Valero V, Lindau M, Alvarez de Toledo $G$ (1999). High calcium concentrations shift the mode of exocytosis to the kiss-and-run mechanism. Nat Cell Biol 1(1): 40-44. doi: $10.1038 / 9012$

14. Fernandez JM, Neher E, Gomperts BD (1984). Capacitance measurements reveal stepwise fusion events in degranulating mast cells. Nature 312(5993): 453-455. doi: 10.1038/312453a0

15. Gopel S, Zhang Q, Eliasson L, Ma XS, Galvanovskis J, Kanno T, Salehi A, Rorsman P (2004). Capacitance measurements of exocytosis in mouse pancreatic alpha-, beta- and delta-cells within intact islets of Langerhans. J Physiol 556(Pt 3): 711-726. doi: 10.1113/jphysiol.2003.059675

16. Tester M, Zorec R (1992). Cytoplasmic calcium stimulates exocytosis in a plant secretory cell. Biophys J 63(3): 864-867. doi: 10.1016/S0006-3495(92)81662-4

17. Weise R, Kreft M, Zorec R, Homann U, Thiel G (2000). Transient and permanent fusion of vesicles in Zea mays coleoptile protoplasts measured in the cell-attached configuration. J Membr Biol 174(1): 15 20. doi: $10.1007 / \mathrm{s} 002320001027$

18. Breckenridge $\amalg$, Almers W (1987). Currents through the fusion pore that forms during exocytosis of a secretory vesicle. Nature 328(6133): 814-817. doi: 10.1038/328814a0

19. Zimmerberg J, Curran M, Cohen FS, Brodwick M (1987). Simultaneous electrical and optical measurements show that membrane fusion precedes secretory granule swelling during exocytosis of beige mouse mast cells. Proc Natl Acad Sci U S A 84(6): 1585-1589. doi: 10.1073/pnas.84.6.1585

20. Thiel G, Kreft M, Zorec R (2009). Rhythmic kinetics of single fusion and fission in a plant cell protoplast. Ann N Y Acad Sci 1152: 1-6. doi: 10.1111/j.1749-6632.2008.03996.x

21. Stenovec M, Kreft M, Poberaj I, Betz WJ, Zorec R (2004). Slow spontaneous secretion from single large dense-core vesicles monitored in neuroendocrine cells. FASEB J 18(11): 1270-1272. doi: 10.1096/fj.03-1397fje

22. Gucek A, Jorgacevski J, Singh P, Geisler C, Lisjak M, Vardjan N, Kreft M, Egner A, Zorec R (2016). Dominant negative SNARE peptides stabilize the fusion pore in a narrow, release-unproductive state. Cell Mol Life Sci 73(19): 3719-3731. doi: 10.1007/s00018-016-2213-2

23. Kreft M, Jorgacevski J, Vardjan N, Zorec R (2016). Unproductive exocytosis. J Neurochem 137(6): 880-889. doi: 10.1111/jnc.13561 
24. Jorgacevski J, Fosnaric M, Vardjan N, Stenovec M, Potokar M, Kreft M, Kralj-Iglic V, Iglic A, Zorec R (2010). Fusion pore stability of peptidergic vesicles. Mol Membr Biol 27(2-3): 65-80. doi: 10.3109/09687681003597104

25. Flasker A, Jorgacevski J, Calejo Al, Kreft M, Zorec R (2013). Vesicle size determines unitary exocytic properties and their sensitivity to sphingosine. Mol Cell Endocrinol 376(1-2): 136-147. doi: 10.1016/j.mce.2013.06.012

26. Jorgacevski J, Potokar M, Grilc S, Kreft M, Liu W, Barclay JW, Bückers J, Medda R, Hell SW, Parpura V, Burgoyne RD, Zorec R (2011). Munc18-1 tuning of vesicle merger and fusion pore properties. J Neurosci 31(24): 9055-9066. doi: 10.1523/JNEUROSCI.0185-11.2011

27. Almers W, Tse FW (1990). Transmitter release from synapses: does a preassembled fusion pore initiate exocytosis? Neuron 4(6): 813-818. doi: 10.1016/0896-6273(90)90134-2

28. Chang CW, Chiang CW, Jackson MB (2017). Fusion pores and their control of neurotransmitter and hormone release. J Gen Physiol 149(3): 301-322. doi: 10.1085/jgp.201611724

29. Han X, Wang CT, Bai J, Chapman ER, Jackson MB (2004). Transmembrane segments of syntaxin line the fusion pore of $\mathrm{Ca} 2+-$ triggered exocytosis. Science 304(5668): 289-292. doi: 10.1126/science.1095801

30. Nanavati C, Markin VS, Oberhauser AF, Fernandez JM (1992). The exocytotic fusion pore modeled as a lipidic pore. Biophys J 63(4): 1118-1132. doi: 10.1016/S0006-3495(92)81679-X

31. Sharma S, Lindau M (2018). The fusion pore, 60 years after the first cartoon. FEBS Lett 592(21):3542-3562. doi: 10.1002/18733468.13160

32. Gasman S, Vitale N (2017). Lipid remodelling in neuroendocrine secretion. Biol Cell 109(11): 381-390. doi: 10.1111/boc.201700030

33. He L, Wu XS, Mohan R, Wu LG (2006). Two modes of fusion pore opening revealed by cell-attached recordings at a synapse. Nature 444(7115): 102-105. doi: 10.1038/nature05250

34. Grabner CP, Moser T (2018). Individual synaptic vesicles mediate stimulated exocytosis from cochlear inner hair cells. Proc Natl Acad Sci U S A 115(50): 12811-12816. doi: 10.1073/pnas.1811814115

35. Klyachko VA, Jackson MB (2002). Capacitance steps and fusion pores of small and large-dense-core vesicles in nerve terminals. Nature 418(6893): 89-92. doi: 10.1038/nature00852

36. Brose N, Brunger A, Cafiso D, Chapman ER, Diao J, Hughson FM, Jackson MB, Jahn R, Lindau M, Ma C, Rizo J, Shin YK, Sollner TH, Tamm $L$, Yoon TY, Zhang Y (2019). Synaptic vesicle fusion: today and beyond. Nat Struct Mol Biol 26(8): 663-668. doi: 10.1038/s41594-019-0277-z

37. NobelPrize. The Nobel Prize in Physiology or Medicine 2013. Available at: https://www.nobelprize.org/prizes/medicine/2013/ summary/. [Accessed: 10.04.2020]

38. Zhao WD, Hamid E, Shin W, Wen PJ, Krystofiak ES, Villarreal SA, Chiang HC, Kachar B, Wu LG (2016). Hemi-fused structure mediates and controls fusion and fission in live cells. Nature 534(7608): 548552. doi: 10.1038/nature18598

39. Fasshauer D, Otto H, Eliason WK, Jahn R, Brunger AT (1997). Structural changes are associated with soluble $\mathrm{N}$-ethylmaleimide-sensitive fusion protein attachment protein receptor complex formation. J Biol Chem 272(44): 28036-28041. doi: 10.1074/jbc.272.44.28036

40. Hanson PI, Heuser JE, Jahn R (1997). Neurotransmitter release four years of SNARE complexes. Curr Opin Neurobiol 7(3): 310-315. doi: 10.1016/s0959-4388(97)80057-8
41. Hastoy B, Clark A, Rorsman P, Lang J (2017). Fusion pore in exocytosis: More than an exit gate? A beta-cell perspective. Cell Calcium 68 : 45-61. doi: 10.1016/j.ceca.2017.10.005

42. Yang ST, Kreutzberger AJB, Lee J, Kiessling V, Tamm LK (2016). The role of cholesterol in membrane fusion. Chem Phys Lipids 199: 136143. doi: 10.1016/j.chemphyslip.2016.05.003

43. Wang N, Kwan C, Gong X, de Chaves EP, Tse A, Tse FW (2010). Influence of cholesterol on catecholamine release from the fusion pore of large dense core chromaffin granules. J Neurosci 30(11): 39043911. doi: 10.1523/JNEUROSCI.4000-09.2010

44. Xu Y, Toomre DK, Bogan JS, Hao M (2017). Excess cholesterol inhibits glucose-stimulated fusion pore dynamics in insulin exocytosis. J Cell Mol Med 21(11): 2950-2962. doi: 10.1111/jcmm.13207

45. Churchward MA, Rogasevskaia T, Brandman DM, Khosravani $H$, Nava P, Atkinson JK, Coorssen JR (2008). Specific lipids supply critical negative spontaneous curvature--an essential component of native Ca2+-triggered membrane fusion. Biophys J 94(10): 3976-3986. doi: 10.1529/biophysj.107.123984

46. Najafinobar N, Mellander LJ, Kurczy ME, Dunevall J, Angerer TB, Fletcher JS, Cans AS (2016). Cholesterol Alters the Dynamics of Release in Protein Independent Cell Models for Exocytosis. Sci Rep 6: 33702. doi: 10.1038/srep33702

47. Stratton BS, Warner JM, Wu Z, Nikolaus J, Wei G, Wagnon E, Baddeley D, Karatekin E, O'Shaughnessy B (2016). Cholesterol Increases the Openness of SNARE-Mediated Flickering Fusion Pores. Biophys J 110(7): 1538-1550. doi: 10.1016/j.bpj.2016.02.019

48. Rituper B, Flasker A, Gucek A, Chowdhury HH, Zorec R (2012). Cholesterol and regulated exocytosis: a requirement for unitary exocytotic events. Cell Calcium 52(3-4): 250-258. doi: 10.1016/j.ceca.2012.05.009

49. Churchward MA, Rogasevskaia T, Hofgen J, Bau J, Coorssen JR (2005). Cholesterol facilitates the native mechanism of Ca2+-triggered membrane fusion. J Cell Sci 118(Pt 20): 4833-4848. doi: 10.1242/jcs.02601

50. Churchward MA, Coorssen JR (2009). Cholesterol, regulated exocytosis and the physiological fusion machine. Biochem J 423(1): 1-14. doi: 10.1042/BJ20090969

51. Julicher F, Lipowsky R (1996). Shape transformations of vesicles with intramembrane domains. Phys Rev E Stat Phys Plasmas Fluids Relat Interdiscip Topics 53(3): 2670-2683. doi: 10.1103/physreve.53.2670

52. Baumgart T, Hess ST, Webb WW (2003). Imaging coexisting fluid domains in biomembrane models coupling curvature and line tension. Nature 425(6960): 821-824. doi: 10.1038/nature02013

53. Kreutzberger AJB, Kiessling V, Stroupe C, Liang B, Preobraschenski J, Ganzella M, Kreutzberger MAB, Nakamoto R, Jahn R, Castle JD, Tamm LK (2019). In vitro fusion of single synaptic and dense core vesicles reproduces key physiological properties. Nat Commun 10(1): 3904. doi: 10.1038/s41467-019-11873-8

54. Kreft M, Kuster V, Grilc S, Rupnik M, Milisav I, Zorec R (2003). Synaptotagmin I increases the probability of vesicle fusion at low [Ca2+] in pituitary cells. Am J Physiol Cell Physiol 284(2): C547-554 doi: 10.1152/ajpcell.00333.2002

55. Lasic E, Stenovec M, Kreft M, Robinson PJ, Zorec R (2017). Dynamin regulates the fusion pore of endo- and exocytotic vesicles as revealed by membrane capacitance measurements. Biochim Biophys Acta 1861(9): 2293-2303. doi: 10.1016/j.bbagen.2017.06.022

56. Rupnik M, Kreft M, Sikdar SK, Grilc S, Romih R, Zupancic G, Martin $\mathrm{TF}$, Zorec $\mathrm{R}$ (2000). Rapid regulated dense-core vesicle exocytosis 
requires the CAPS protein. Proc Natl Acad Sci U S A 97(10): 56275632. doi: 10.1073/pnas.090359097

57. Aunis D, Bader MF (1988). The cytoskeleton as a barrier to exocytosis in secretory cells. J Exp Biol 139: 253-266. PMID: 3062121

58. Chowdhury HH, Kreft M, Zorec R (2002). Distinct effect of actin cytoskeleton disassembly on exo- and endocytic events in a membrane patch of rat melanotrophs. J Physiol 545(Pt 3): 879-886. doi: 10.1113/jphysiol.2002.028043

59. Chowdhury HH, Popoff MR, Zorec R (2000). Actin cytoskeleton and exocytosis in rat melanotrophs. Pflugers Arch 439(3 Suppl): R148-149. doi: $10.1007 /$ s004240000125

60. Burgoyne RD, Morgan A (2003). Secretory granule exocytosis. Physiol Rev 83(2): 581-632. doi: 10.1152/physrev.00031.2002

61. Chiang HC, Shin W, Zhao WD, Hamid E, Sheng J, Baydyuk M, Wen PJ, Jin A, Momboisse F, Wu LG (2014). Post-fusion structural changes and their roles in exocytosis and endocytosis of dense-core vesicles. Nat Commun 5: 3356. doi: 10.1038/ncomms4356

62. Wen PJ, Grenklo S, Arpino G, Tan X, Liao HS, Heureaux J, Peng SY, Chiang HC, Hamid E, Zhao WD, Shin W, Nareoja T, Evergren E, Jin $Y$, Karlsson R, Ebert SN, Jin A, Liu AP, Shupliakov O, Wu LG (2016). Actin dynamics provides membrane tension to merge fusing vesicles into the plasma membrane. Nat Commun 7: 12604 . doi: $10.1038 /$ ncomms 12604

63. Shin W, Arpino G, Thiyagarajan S, Su R, Ge L, McDargh Z, Guo X, Wei L, Shupliakov O, Jin A, O'Shaughnessy B, Wu LG (2020). Vesicle Shrinking and Enlargement Play Opposing Roles in the Release of Exocytotic Contents. Cell Rep 30(2): 421-431 e427. doi: 10.1016/j.celrep.2019.12.044

64. Stenmark H (2009). Rab GTPases as coordinators of vesicle traffic. Nat Rev Mol Cell Biol 10(8): 513-525. doi: 10.1038/nrm2728

65. Montana V, Liu W, Mohideen U, Parpura V (2009). Single molecule measurements of mechanical interactions within ternary SNARE complexes and dynamics of their disassembly: SNAP25 vs. SNAP23. J Physiol 587(Pt 9): 1943-1960. doi: 10.1113/jphysiol.2009.168575
66. Coorssen JR, Zorec R (2012). Regulated exocytosis per partes. Cell Calcium 52(3-4): 191-195. doi: 10.1016/j.ceca.2012.06.003

67. Pascual O (2005). Astrocytic purinergic signaling coordinates synaptic networks. Science 310: 113-116. doi: 10.1126/science.1116916

68. Verkhratsky A, Matteoli M, Parpura V, Mothet JP, Zorec R (2016). Astrocytes as secretory cells of the central nervous system: idiosyncrasies of vesicular secretion. EMBO J 35(3):239-57. doi: 10.15252/embj.201592705

69. Kreft M, Stenovec M, Rupnik M, Grilc S, Krzan M, Potokar M, Pangrsic T, Haydon PG, Zorec R (2004). Properties of $\mathrm{Ca}(2+)$ dependent exocytosis in cultured astrocytes. Glia 46(4): 437-445. doi: 10.1002/glia.20018

70. Baneux C, Tanguy E, Thahouly T, Vitale A, Chasserot-Golaz S, Bader MF, Gasman S, Vitale N (2020). Phosphatidic acid metabolism regulates neuroendocrine secretion but is not under the direct control of lipins. IUBMB Life 72(4): 533-543. doi: 10.1002/iub.2229

71. Kolay S, Basu U, Raghu P (2016). Control of diverse subcellular processes by a single multi-functional lipid phosphatidylinositol 4,5bisphosphate [PI(4,5)P2]. Biochem J 473(12): 1681-1692. doi: 10.1042/BCJ20160069

72. Rituper B, Guček, A., Lisjak, M., Gorska, U., Šakanović, A., Trkov Bobnar, S., Lasič, E., Božić, B., Abbineni, P.S., Jorgačevski, J., Kreft, M. Verkhratsky, A., Platt, F. M., Anderluh, G., Stenovec, M., Božič, B., Coorssen, J. R., Zorec, R. (2020). Redistribution of cholesterol from vesicle to plasmalemma controls fusion pore geometry. Biorxiv. doi: 10.1101/2020.04.06.027169

73. Del Castillo J, Katz B (1957). La base "quantale" de la transmission neuomusculaire. In: Microphysiologie Comparee des Elements Excitables. Coll Int CNRS 67: 245-258.

74. Calejo Al, Jorgacevski J, Rituper B, Gucek A, Pereira PM, Santos MA, Potokar M, Vardjan N, Kreft M, Goncalves PP, Zorec R (2014). Hyperpolarization-activated cyclic nucleotide-gated channels and cAMP-dependent modulation of exocytosis in cultured rat lactotrophs. J Neurosci 34(47): 15638-15647. doi: 10.1523/JNEUROSCI.529013.2014 